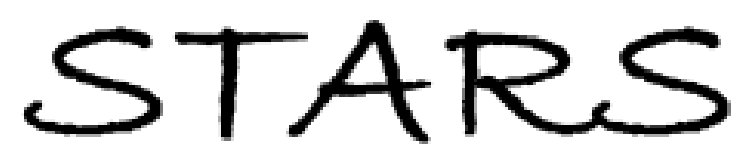

University of Central Florida

STARS

Faculty Bibliography 2000s

Faculty Bibliography

$1-1-2003$

\title{
Lattice solitons in Bose-Einstein condensates
}

Nikolaos K. Efremidis

University of Central Florida

Demetrios N. Christodoulides

University of Central Florida

Find similar works at: https://stars.library.ucf.edu/facultybib2000

University of Central Florida Libraries http://library.ucf.edu

This Article is brought to you for free and open access by the Faculty Bibliography at STARS. It has been accepted for inclusion in Faculty Bibliography 2000 s by an authorized administrator of STARS. For more information, please contact STARS@ucf.edu.

\section{Recommended Citation}

Efremidis, Nikolaos K. and Christodoulides, Demetrios N., "Lattice solitons in Bose-Einstein condensates" (2003). Faculty Bibliography 2000s. 3728.

https://stars.library.ucf.edu/facultybib2000/3728

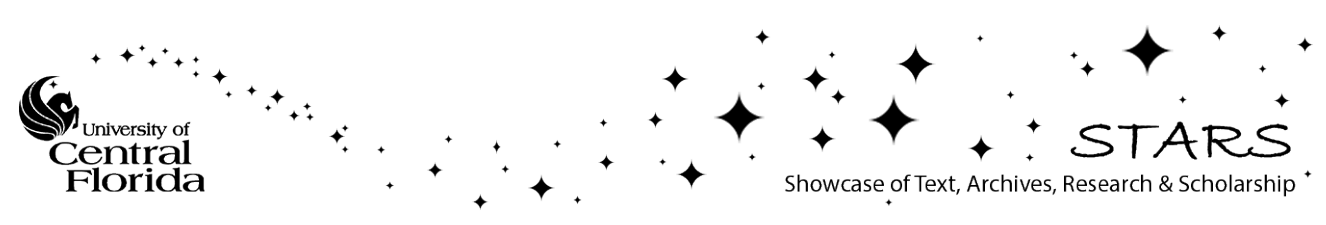




\title{
Lattice solitons in Bose-Einstein condensates
}

\author{
Nikolaos K. Efremidis and Demetrios N. Christodoulides \\ Center for Research and Education in Optics and Lasers/School of Optics, University of Central Florida, Orlando, Florida 32816, USA
}

(Received 17 January 2003; published 20 June 2003)

\begin{abstract}
We systematically study the properties of lattice solitons in Bose-Einstein condensates with either attractive or repulsive atom interactions. This is done, by exactly solving the mean-field Gross-Pitaevskii equation in the presence of a periodic potential. We find new families of lattice soliton solutions that are characterized by the position of the energy eigenvalue within the associated band structure. These include lattice solitons in condensates with either attractive or repulsive atom interactions that exist in finite or semi-infinite gaps, as well as nonlinear modes that exhibit atomic population cutoffs.
\end{abstract}

DOI: 10.1103/PhysRevA.67.063608

PACS number(s): 03.75.Lm, 05.45.Yv

\section{INTRODUCTION}

In recent experiments Bose-Einstein condensates (BEC) have been successfully loaded in optical lattices $[1,2]$ and, ever since, this field has attracted considerable attention. Subsequently, lattice effects, such as squeezed states [3], Bloch oscillations [4], Josephson-junction arrays [5], superfluid and dissipative dynamics [6], dispersion [7,8], Talbot effects [9], and Landau-Zener tunneling [10] have been experimentally demonstrated. In addition to that, several theoretical works have investigated the linear properties of such lattices $[11,12]$.

In these experiments, carried out at ultralow temperatures, the wave function of the condensed atoms obeys a GrossPitaevskii equation which is mathematically equivalent to the so-called nonlinear Schrödinger equation. The optical lattice involved is created by the interference of laser beams, and the properties of the atoms are characterized by the depth and period of this optically induced potential. When the periodic potential wells are deep enough, the atom density tends to increase at the potential minima, thus creating, essentially, an array of optical traps. Because of proximity, atoms can tunnel between adjacent traps and, as a result, they become quantum mechanically coupled. In principle, if the BEC atoms are loaded on one site, the condensate is expected to disperse in time to all the other sites.

In the regime where the atomic density is high, the condensate behaves nonlinearly, giving rise to a host of different phenomena [13-19]. An interesting manifestation of the nonlinearity is the existence of self-localized BEC states or lattice solitons $[13,14]$. These entities are possible when the nonlinearity compensates for atom dispersion caused by intersite tunneling. These lattice solitons are characterized by the position of their eigenvalue within the $E-k$ band structure. In the case where only the first band is considered and the potentials happen to be deep enough, these self-localized states are better known as discrete solitons simply because they can be described by the tight-binding approximation. On the other hand, when the eigenenergy is located in the gap between two successive bands, these self-localized states are known as gap solitons [20,21] and, for relatively shallow potentials, are described by coupled-mode equations [22]. Here, we will use the term lattice soliton to generally describe all the families of self-localized solutions that exist inside a periodic potential (or lattice). Experimentally, discrete solitons have been first demonstrated in self-focusing arrays of nonlinear waveguides [23] etched onto an $\mathrm{Al}_{x} \mathrm{Ga}_{1-x} \mathrm{As}$ substrate in one dimension [24]. Recently, using optical induction techniques [25], both self-focusing and self-defocusing discrete solitons have been observed in onedimension $[26,27]$ and, subsequently, for the first time, in two-dimensions [28].

In general, the validity of the approximate descriptions used to describe lattice solitons depends heavily on the nature of the underlying problem. For example, the tightbinding approximation is only accurate when the wave function is highly confined into the potential minima, i.e., when the potentials are deep and well separated. In addition, it cannot account for a second band, thus, limiting its accuracy close to the edge of the first Brillouin zone. Similarly, the coupled-mode theory used to describe gap solitons is valid when the energies are close to the gap and for shallow potentials. Strictly speaking, an accurate solution can only be obtained by exactly solving the full nonlinear Schrödinger equation with a periodic potential. In Ref. [25], such lattice solitons that exist at the base and edge of the first Brillouin zone were found for both one- and two-dimensional crystals by numerically solving a saturable nonlinear Schrödinger equation. In the same work, it was also shown that the discrete model fails to predict the transport properties of lattice (gap) solitons at the edge of the first Brillouin zone. In an interesting work, Mandelik et al. [29] demonstrated the existence of nonlinear Floquet-Bloch modes in nonlinear waveguide arrays. Finally, Louis et al. [30] predicted the existence of families of spatially localized matter-wave gap solitons and analyzed their stability.

Here, we use a general approach to identify lattice solitons in BEC with both attractive and repulsive atomic interactions. Our analysis is based on the solution of the meanfield nonlinear Schrödinger equation with a periodic potential. Such a model was first used in Ref. [25] to identify lattice soliton solutions in saturable media. Two types of lattice solitons were found in that paper: self-focusing discrete solitons, residing on the semi-infinite band gap as well as self-defocusing lattice solitons with eigenvalues in the first band gap. In Ref. [30] the families of these lattice solitons (i.e., gap modes for repulsive condensates and lattice soliton on the semi-infinite band gap for attractive condensates) 
were obtained and characterized according to their position inside the band structure.

In this paper we systematically study lattice solitons in Bose-Einstein condensates. New families of lattice solitons are found and classified according to their position inside the band structure. More specifically, when the eigenvalue is located in the semi-infinite gap, we show that, except from the so-called discrete solitons, other classes of nonlinear modes that exhibit atomic population cutoffs and cannot be predicted by the tight-binding approximation, can exist. When the eigenvalue lies inside a band gap, gap lattice solitons are found for repulsive as well as for attractive condensates. It is shown that close to the first band the gap modes become relatively broad and their wave function can be approximated by the Floquet-Bloch mode of the corresponding band. On the other hand, when the eigenvalue is close to the second band, these modes become highly confined inside the lattice (exhibiting a cusplike behavior). Finally, we compare the results found here with those obtained from approximate procedures based on the tight-binding approximation or the coupled-mode theory.

\section{BOSE-EINSTEIN CONDENSATES INSIDE A LATTICE}

The evolution of the mean-field wave function $\Psi$ of a Bose-Einstein condensate in an optical trap obeys the GrossPitaevskii equation [31,32]

$$
i \hbar \frac{\partial \Psi}{\partial t}=-\frac{\hbar^{2}}{2 m} \nabla^{2} \Psi+V(\mathbf{r}) \Psi+g|\Psi|^{2} \Psi,
$$

where $\hbar$ is Planck's constant, $m$ is the atomic mass, $g$ $=4 \pi a_{s} \hbar^{2} / m$ is the nonlinear coefficient that takes into account the mean field produced by the other bosons, and $a_{s}$ is the $s$-wave scattering length. In Eq. (1),

$$
V(\mathbf{r})=E_{0} \sin ^{2}\left(\frac{\pi x}{L}\right)+\frac{1}{2} m\left[\omega_{x}^{2} x^{2}+\omega_{\perp}^{2}\left(y^{2}+z^{2}\right)\right]
$$

describes both the trap potential and that arising from the periodic, optically induced, interference pattern. In Eq. (2), $\omega_{x}$ and $\omega_{\perp}$ are the trap frequencies, $L$ is the lattice period, $m$ is the mass of the atoms, and $E_{0}$ is the potential depth (usually measured with respect to the recoil energy). The trap is elongated along the $x$ direction (i.e., $\omega_{\perp} \gg \omega_{x}$ ). If we express the wave function as $\Psi(x, y, z ; t)=U(y, z) \psi(x, t)$, and decompose the potential as $V(x, y, z)=V_{x}(x)+V_{\perp}(y, z)$ then, due to the high confinement in the $y-z$ plane, $U$ approximately satisfies

$$
-\frac{\hbar^{2}}{2 m} \nabla_{\perp}^{2} U+\frac{1}{2} m \omega_{\perp}^{2} \rho^{2} U=E_{\perp} U .
$$

Equation (3) can be solved analytically and its first (zeronode) eigenfunction is given by

$$
U=\sqrt{\frac{m \omega_{\perp}}{\pi \hbar}} \exp \left[-\frac{m \omega_{\perp}}{2 \hbar}\left(y^{2}+z^{2}\right)\right],
$$

with an eigenvalue $E_{\perp}=\hbar \omega_{\perp}$. By integrating in the $y-z$ plane, applying the transformation $\psi \rightarrow \psi \exp \left(-i \omega_{\perp} t\right)$, and assuming that the frequency of the trap $\left(\omega_{x}\right)$ is much bigger than the lattice frequency $2 \pi / L$, one can obtain

$$
i \hbar \frac{\partial \psi}{\partial t}=-\frac{\hbar^{2}}{2 m} \psi_{x x}+E_{0} \sin ^{2}(\pi x / L) \psi+2 \hbar \omega_{\perp} a_{s}|\psi|^{2} \psi .
$$

It is more convenient to use dimensionless quantities by normalizing $T=t / T_{0}, \quad X=x /(L / 2), \quad \psi=u / L_{1}^{1 / 2}, \quad$ and $V_{0}$ $=E_{0} / E_{r}$, and choose $T_{0}=m L^{2} / 4 \hbar, L_{1}=\omega_{\perp}\left|a_{s}\right| m L^{2} / 2 \hbar$, and $E_{r}=4 \hbar^{2} / m L^{2}$. After these transformations,

$$
i \frac{\partial u}{\partial T}=-\frac{1}{2} \frac{\partial^{2} u}{\partial X^{2}}+V_{0} \sin ^{2}\left(\frac{\pi x}{2}\right) u+\sigma|u|^{2} u,
$$

where $\sigma=\operatorname{sgn}\left(a_{s}\right)$. In recent BEC experiments in lattices, ${ }^{87} \mathrm{Rb}$ atoms were used to produce the condensate. For ${ }^{87} \mathrm{Rb}$ atoms, $m=1.4 \times 10^{-25} \mathrm{~kg}$, while the $s$-scattering length $a_{s}$ $=5.77 \mathrm{~nm}$ is positive, resulting in repulsive interactions. Typical values of the lattice spacing can vary between 0.4 and $1.6 \mu \mathrm{m}$, whereas the lattice depth (normalized to $E_{r}$ ) can be $V_{0} \lesssim 22$. Here, we assume a lattice spacing of $1 \mu \mathrm{m}$ and a normalized potential depth $V_{0}=10$, unless stated otherwise. Attractive nonlinearities are also possible, for example, for ${ }^{7} \mathrm{Li}$ atoms $\left(m=0.115 \times 10^{-25} \mathrm{~kg}, a_{s}\right.$ $=-1.457 \mathrm{~nm}$ ). We would like to mention that Eq. (6) possesses an integral of motion,

$$
N=\int_{-\infty}^{\infty}|u|^{2} d X
$$

which, physically, accounts for the conservation of the number of atoms in the condensate.

\section{BAND STRUCTURE}

The $E-k$ band structure of the lattice interacting with the condensate plays an important role, since it determines basic properties of the matter waves under linear conditions. To find the band structure we assume that the linear part of Eq. (6) admits solutions of the form $u=v \exp (-i E T)$, thus obtaining the following eigenvalue problem:

$$
E v=-\frac{1}{2} \frac{\partial^{2} v}{\partial X^{2}}+V_{0} \sin ^{2}\left(\frac{\pi X}{2}\right) v .
$$

Over the years several methods have been developed to solve such eigenvalue problems. Here, we use the plane-wave method that provides a generic algorithm for periodic potentials [33] (see the Appendix). A typical band structure of Eq. (8) for $V_{0}=10$ and $L=1 \mu \mathrm{m}$ is shown in Fig. 1. For values of $E$ inside the energy bands, Eq. (8) possesses periodic solutions, better know as Floquet-Bloch (FB) modes. According to Bloch's theorem, the Floquet-Bloch modes, i.e., the eigenfunctions of Eq. (8), are characterized by their momentum $k$ inside the lattice. More specifically, a FB mode with momentum $k$ can be expressed as $v_{k}(X)=V_{k}(X) \exp (i k X)$, 


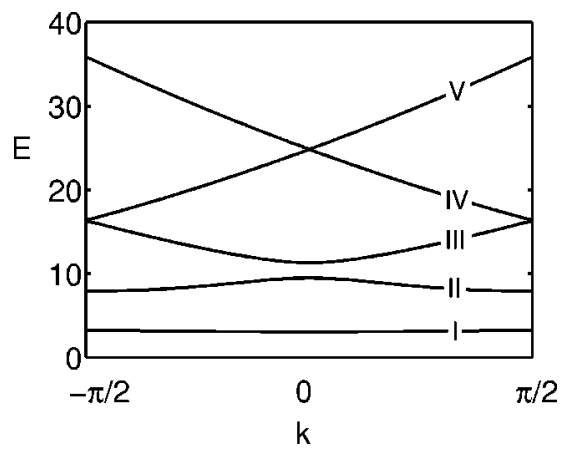

FIG. 1. A typical band structure for $V_{0}=10$ and $L=1 \mu \mathrm{m}$. Curves I-V correspond to the first five bands, respectively. For this example, the band gap between bands III and IV, and IV and V is very small.

where $V_{k}(X)$ is a periodic function with the period of the lattice. Notice that at the base and the edge of each band $(k=0, \pi / 2)$ the eigenfunctions $v_{k}(X)$ are always real. In Fig. 2 such modes located at the base and the edge of the Brillouin zone are depicted for the first four bands. At the base of the Brillouin zone, and since $\exp (i k X)=\exp [i k(X+2)], v_{k}$ will have the period of the lattice. At the edge of the zone, $\exp (i k X)=-\exp [i k(X+2)]$, and thus, $v_{k}$ will have period 4 (i.e., twice the period of the lattice). Particular nonlinear FB modes have been recently demonstrated in both one and two dimensions [15-17]. According to their momentum, these modes can be modulationally stable or unstable.

Inside the band gaps, exponentially decaying modes also exist. These modes can be obtained by using, for example, the plane-wave method, where now the lattice momentum is allowed to be complex. Approximate expressions for these modes and their rate of decay inside the first band gap can be found by keeping only the first-order resonant terms in the plane-wave expansion (see the Appendix for details) as long as the potentials are not very deep. Along these lines, one finds that the energy at the edge of the first band is $\pi^{2} / 8$ $+V_{0} / 4$, whereas at the edge of the second is $\pi^{2} / 8+3 V_{0} / 4$, and thus the energy band gap is $V_{0} / 2$. Furthermore, the decay rate of these modes inside the band gap is given by

$$
k_{i}^{2}=-2 E-\frac{\pi^{2}}{4}+V_{0}+\pi \sqrt{2 E-V_{0}+\left(V_{0} / \pi\right)^{2}} .
$$

As we will see later, Eq. (9) also describes the rate of decay of the soliton tails at eigenvalue $E$. This should have been anticipated since the low-amplitude tails can be accurately described in the linear regime. From Eq. (9) it is easy to see that when $E$ is close to one of the bands, $k_{i}$ becomes very small and the soliton tails decay very slowly at $\pm \infty$. The fastest decay is achieved close to the center of the band gap (when $V_{0}$ is small), and more specifically for $E=\pi^{2} / 8$ $+V_{0} / 2-V_{0}^{2} / 8 \pi^{2}$. The decaying modes inside the band gap are then given by

$$
v=\left[\cos \frac{\phi}{2} \cos \frac{\pi X}{2}+\sin \frac{\phi}{2} \sin \frac{\pi X}{2}\right] e^{-k_{i} x},
$$
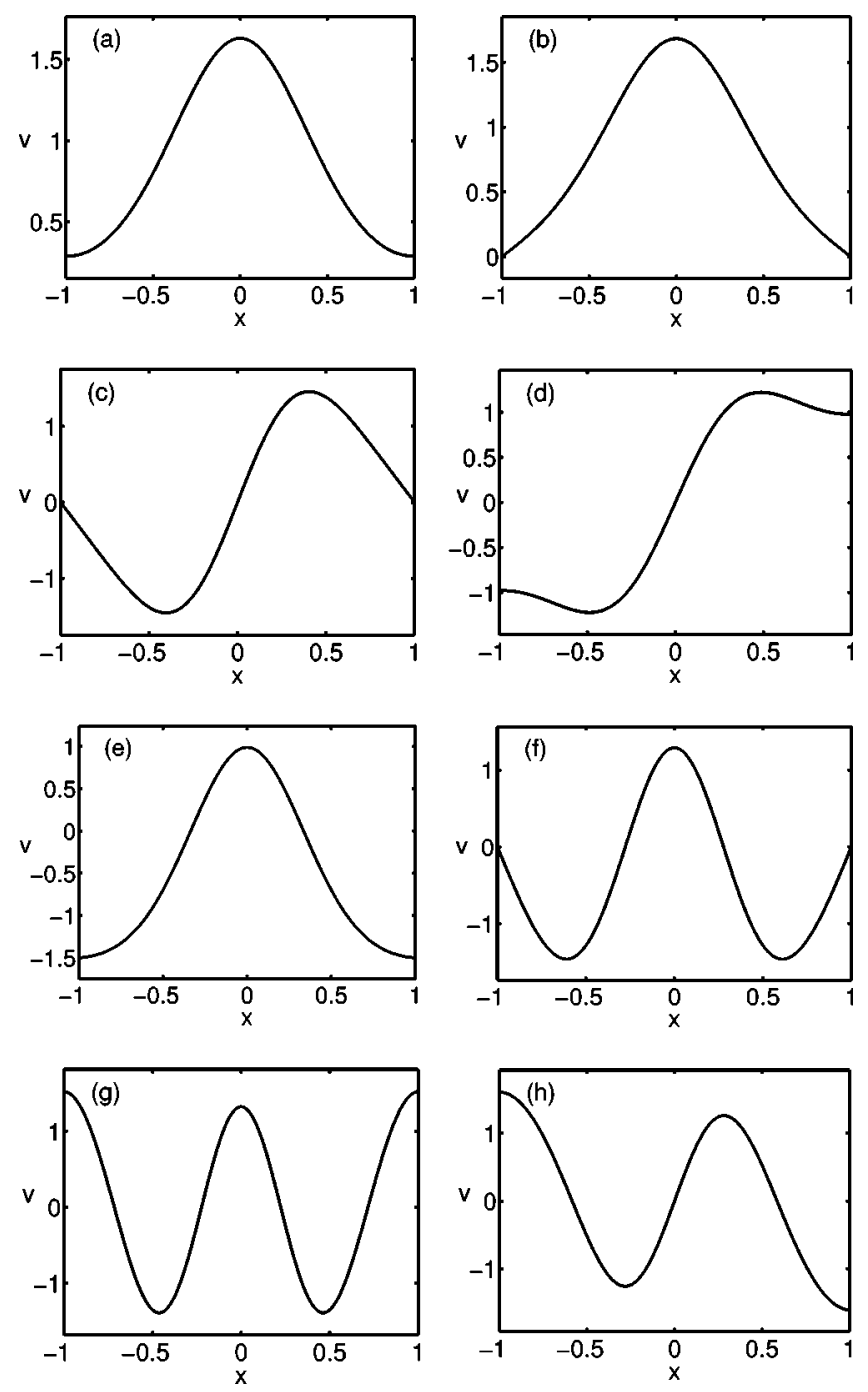

FIG. 2. The linear Floquet-Bloch modes for $V_{0}=10$ and $L$ $=1 \mu \mathrm{m}$. The left (right) column corresponds to the base (edge) of the Brillouin zone. The four rows (from top to bottom) correspond to the first four bands, respectively.

where $\phi=\arctan (\beta / \alpha)$ and $\alpha=\pi^{2} / 8+V_{0} / 2-k_{i}^{2} / 2-E, \quad \beta$ $=k_{i} \pi / 2$. Similar expressions can also be obtained for eigenvalues below the first band (semi-infinite gap). At the edges of the first band gap, i.e., inside the first and the second band when $k=\pi / 2$, the Floquet-Bloch mode are approximately given by $\cos (\pi X / 2)$ and $\sin (\pi X / 2)$, respectively. Equation (10) is a product of an exponentially decaying function with a periodic function in $X$. The periodic part is a linear superposition of the Floquet-Bloch modes at the edges of the two bands. The closer the eigenvalue is to one band, the more this periodic function will look like the corresponding Floquet-Bloch mode (see the Appendix).

\section{LATTICE SOLITONS}

Immobile lattice solitons can only be found within the gaps as nonlinear defect modes. To find such self-localized states, we assume that Eq. (6) admits solitons of the form $u(x) \exp (-i E T)$, and thus, 


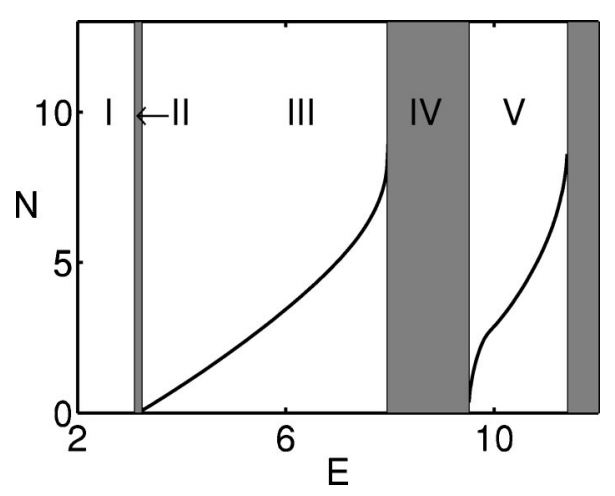

FIG. 3. Number of atoms, $N$, as a function of the eigenvalue $E$, for repulsive condensates with $V_{0}=10$ and $L=1 \mu \mathrm{m}$. Regions I, III, and V correspond to the semiinfinite gap and the first two band gaps, whereas, the shaded regions II and IV depict the first two bands, respectively.

$$
E u=-\frac{1}{2} \frac{\partial^{2} u}{\partial X^{2}}+V_{0} \sin ^{2}\left(\frac{\pi x}{2}\right) u+\sigma u^{3}
$$

Equation (11) is then solved numerically. These selflocalized states are characterized by the position of their eigenenergies inside the gaps. Physically, the nonlinearity provides the potential necessary to "connect" the two exponentially decaying modes to a homoclinic orbit. We found that lattice solitons exist for both attractive and repulsive atom interactions.

\section{A. Repulsive interactions}

When the nonlinearity is repulsive, lattice solitons do not exist below the first band shown in Fig. 1. This is because the effective dispersion at the base of the first Brillouin zone is positive or normal. On the other hand, in the same regime (repulsive) lattice solitons can exist inside the band gaps of Eq. (8). We have isolated the first two families of these lattice solitons. In Fig. 3, the normalized atom number $N$ of these solutions is depicted as a function of the eigenvalue. When $E$ approaches the left edge $E_{L, j}$ of the $j$ th band gap (of Fig. 3), $N$ goes to zero, whereas close to the right edge $E_{R, j}$ of the band gaps, the solutions become highly nonlinear. Qualitatively, this can be explained by looking at the effective dispersion inside the band structure. When $E$ approaches $E_{L, j}$, the dispersion is anomalous and, thus, broad (lowamplitude) lattice solitons can be supported even for small values of the nonlinearity. On the other hand, since the dispersion on the right side of each band gap is normal, a large amount of nonlinearity is required to form a lattice soliton. Considering that close to $E_{R, j}$, the tails of the solution will decay slowly, the envelope of the lattice soliton will exhibit a cusplike behavior.

In Fig. 4 typical lattice solitons in repulsive condensates are depicted. Close to the edge of the first Brillouin zone [Fig. 4(a)], the maximum atomic density $|u|^{2}$, as well as $N$, are very small. As we can see, the form of the soliton can be approximated by the corresponding Floquet-Bloch mode [see Fig. 2(b)] multiplied by an envelope function. This envelope
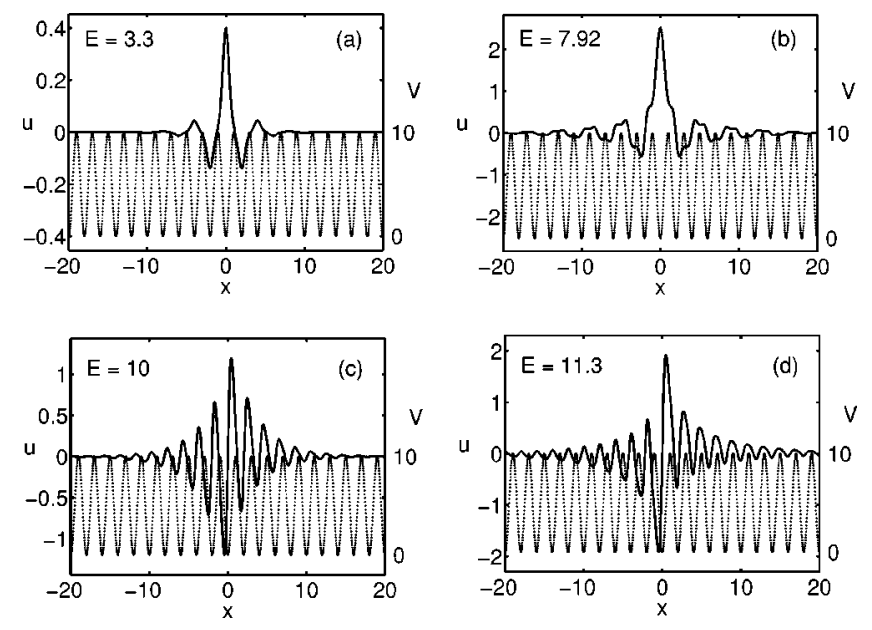

FIG. 4. Lattice solitons in repulsive condensates. The first and the second row depict solitons in the first and second band gaps, respectively. The left (right) column shows typical soliton solutions with energies close to $E_{L, j}\left(E_{R, j}\right)$ for $j=1,2$.

is broad (occupying many lattice sites) and decay slowly at $\pm \infty$ according to Eq. (9). Going deeper inside the band gap, the solitons become more and more localized inside the lattice, and their tails can be expressed as a superposition of the Floquet-Bloch modes at the edges of the first band gap. As it can be seen in Fig. 4(b), when the eigenvalue gets close to the edge of the second Brillouin zone, the form of the soliton tails can be described by the corresponding Floquet-Bloch mode. This becomes obvious by comparing the tails of the soliton solution of Fig. 4(b) with Fig. 2(d). Furthermore, since from Eq. (9) $k_{i} \rightarrow 0$ when $E \rightarrow E_{R, j}$, the soliton tails decay very slowly. The behavior of the oscillations of the lattice soliton at its peak atom density is very much different from that at its tails, and cannot be described by the corresponding Floquet-Bloch mode. Physically, this happens because close to the edge of the second band the effective diffraction is mainly affected by the second band (and much less by the first band). As a result, a high degree of nonlinearity is required to support a lattice soliton (that will now have a cusplike envelope). Using similar arguments, one can describe the lattice solitons in the second band gap [Figs. 4(c) and 4(d)] (between the edge of the second and the third Brillouin zones). Similar solutions can be found inside the $n$th band gap, although the width of each band gap decreases with increasing $n$.

\section{B. Attractive interactions}

In the case of condensates with attractive interactions, we have also found the first three families of lattice solitons. In Fig. 5 the number of atoms vs the eigenvalue is depicted when $V_{0}=10$ and $L=1 \mu \mathrm{m}$. The first family of lattice solitons exists in the semi-infinite gap that can be found below the first band. Such solitons can exist as a result of the normal effective dispersion at the base of the first band. In this case, the band gap is semi-infinite, and thus, the form of the soliton tails can always be approximated by the FB mode at the base of the first zone multiplied by an exponentially decaying $\exp \left(-k_{i} x\right)$ amplitude, where $k_{i}$ increases by going 


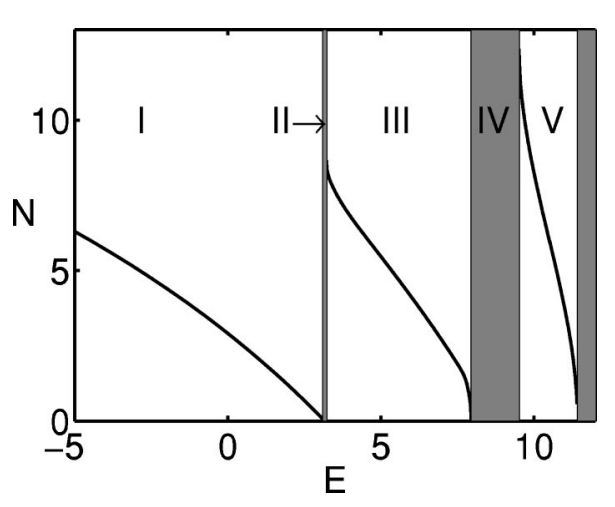

FIG. 5. Number of atoms, $N$, as a function of the eigenvalue $E$, for attractive atom interactions. Regions I, III, and V correspond to the semi-infinite gap and the first two band gaps, whereas the shaded regions II and IV depict the first two bands, respectively.

deeper into the gap. Thus, the solutions become narrower as the eigenvalue $E$ decreases. In Fig. 6 such a lattice soliton is depicted. In this case (i.e., $V_{0}=10$ and $L=1 \mu \mathrm{m}$ ), there is clearly a local maximum in the atom density at each potential minimum.

Lattice solitons in attractive condensates can also exist inside the band gaps. In Fig. 7 such self-localized solutions are depicted. The properties of these solutions can be qualitatively described by using similar arguments. In contrast to the repulsive case, now $N$ becomes small on the right side of each band gap of Fig. 5, whereas, the lattice solitons become highly nonlinear on the left side of each gap. Close to the edge of the second Brillouin zone the effective lattice dispersion is normal; the solutions become broad occupying many lattice sites, and can be approximated by the FB mode at the edge of the second Brillouin zone shown in Fig. 2(d). As the eigenvalue decreases inside the first band gap, the FloquetBloch modes become mixed and the lattice soliton becomes more localized inside the lattice. Finally, close to the edge of the first band, the effective diffraction becomes anomalous and a highly nonlinear, cusplike mode is required to support a lattice soliton [Fig. 7(a)]. Figures 7(c) and 7(d) show typical lattice solitons in the second band gap with eigenvalues close to the base of the second and the third bands, respectively.

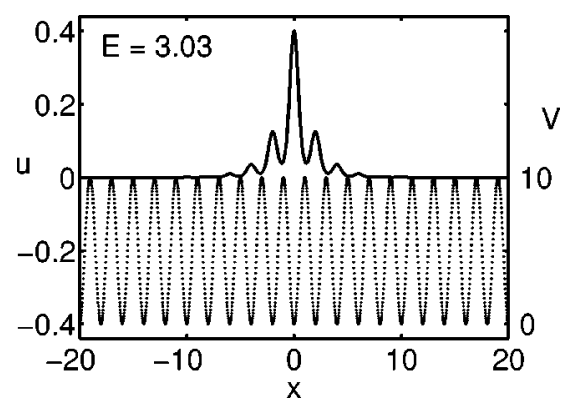

FIG. 6. A typical form of a lattice soliton (solid curve) in attractive condensates with eigenvalue in the gap below the first band for $v_{0}=10$ and $L=1 \mu \mathrm{m}$. The dotted curve represents the periodic lattice potential $V$.
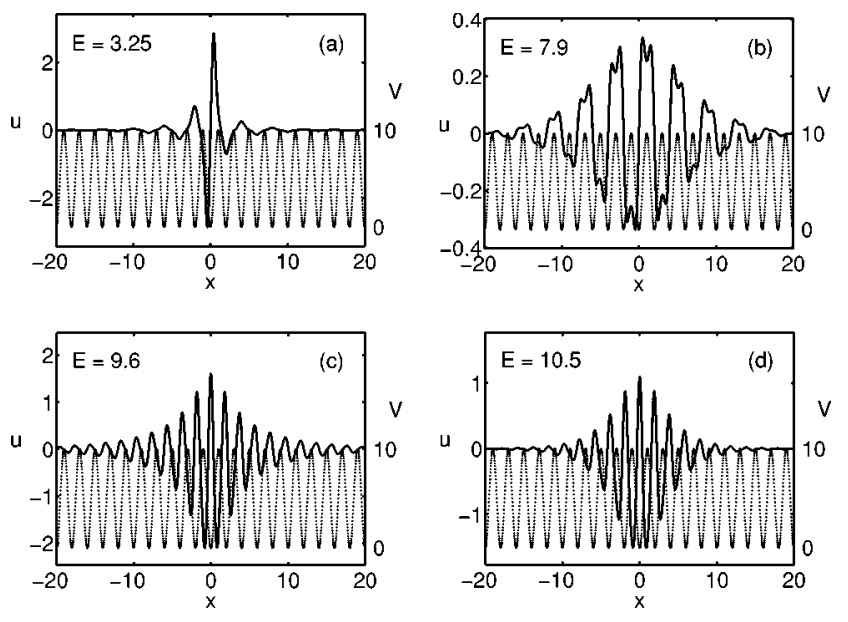

FIG. 7. Lattice solitons (solid curves) for attractive atom interactions when $V_{0}=10$ and $L=1 \mu \mathrm{m}$. The first (second) row depicts lattice solitons in the first (second) band, whereas the left (right) column corresponds to the left (right) edge of each band gap in Fig. 5. The dotted curves represent the periodic lattice potential $V$.

\section{Nonlinear modes with a cutoff}

The families of lattice solitons previously described have a common property. In one limit (close to one band) the number of atoms $(N)$ approaches zero, whereas in the other limit (close to the second band or for very small values of $E$ when the gap is semi-infinite), these solitons become highly nonlinear. Except these families, we were able to isolate additional families that represent new types of nonlinear modes with atomic population cutoffs, i.e., the number of atoms $(N)$ never goes to zero, but, instead, exhibits a threshold value $N_{0}$. These modes can either exist in the semi-infinite gap or inside finite band gaps. The existence of this type of lattice solitons can be qualitatively understood as follows. The nonlinearity is responsible for an effective potential $|u|^{2}$ that modifies the lattice potential that the atoms experience. After a certain threshold additional bound modes form, which, in turn, allow additional higher order lattice solitons in the array. In Fig. 8 the $N-E$ diagram of the three first nonlinear odd modes with eigenvalues inside the semi-infinite band gap is

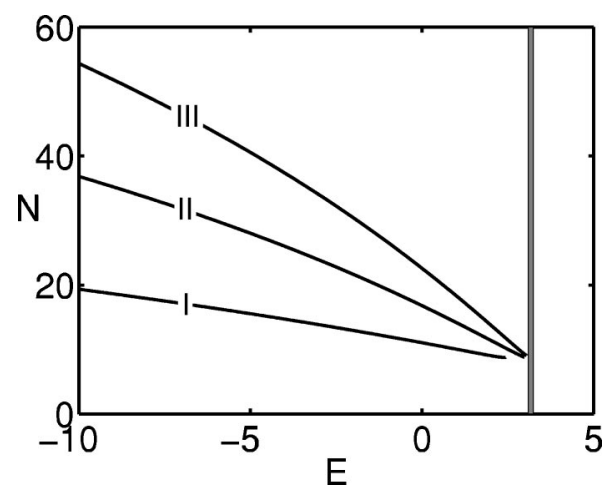

FIG. 8. Number of atoms, $N$, as a function of the eigenvalue $E$ for the first three even lattice solitons with a cutoff. The vertical shaded area shows the width of the first Brillouin zone. Branches I-III correspond to the first three modes, respectively. 

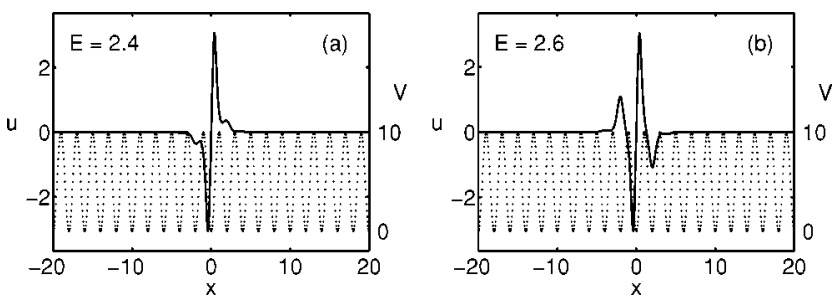

FIG. 9. Typical field profiles of the first two odd lattice solitons with a cutoff in attractive condensates.

shown. Typical odd soliton profiles are depicted in Fig. 9. Note that in these solutions the eigenfunction goes to zero and has two peaks at the center site-a behavior that cannot be accounted within the tight-binding approximation. Even nonlinear modes with a cutoff are also shown in Fig. 10. We would like to mention that this type of nonlinear lattice solitons with a cutoff cannot be predicted by the tight-binding approximation because the original expansion is not complete, accounting only for solutions localized in the lattice minima.

\section{Stability}

We have performed a series of numerical simulations to test the stability of these solutions. A random perturbation was added to the exact solution at $T=0$ to make sure that all the linear perturbation eigenmodes are exited. Using a splitstep Fourier method, we numerically solve Eq. (6) and monitor the time evolution of the BEC lattice soliton. We have found that the lattice soliton that resides below the first band is always stable (i.e., the atom density $|u|^{2}$ does not change with $T$ ). The solitons that reside in the band gaps can be either stable or unstable depending on their eigenenergies. In general, we found that the lattice solitons are stable in a region close to the band, which allows broad lattice soliton solutions. Going deeper into the band gap, the solutions become narrower, and after a certain threshold they become unstable. In Fig. 11 typical evolution of lattice solitons with eigenenergies in the second band gap are depicted for ${ }^{87} \mathrm{Rb}$ atoms. The eigenenergy of the lattice soliton of Fig. 11(a) is close to the edge of the second band, $E=9.55$, and propagates without any change for over $3 \mathrm{sec}$. On the other hand, the soliton of Fig. 11(b) with eigenvalue $E=10$ turns out to be is unstable.

\section{COMPARISON WITH THE RESULTS OF THE TIGHT-BINDING APPROXIMATION AND COUPLED-MODE THEORY}

To complete our discussion, it may be useful to discuss our results within the context of approximate theories. Sev-
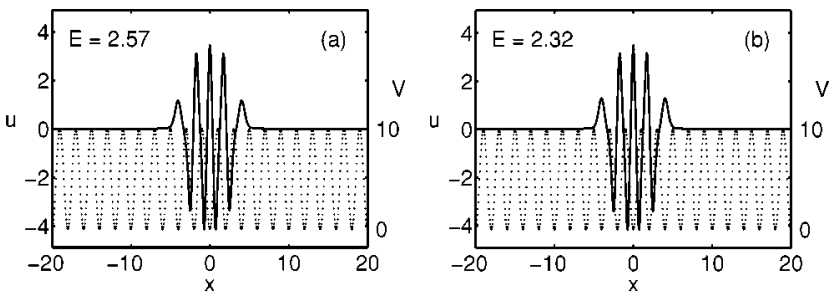

FIG. 10. Typical field profiles of even lattice solitons with a cutoff in attractive condensates. (a)

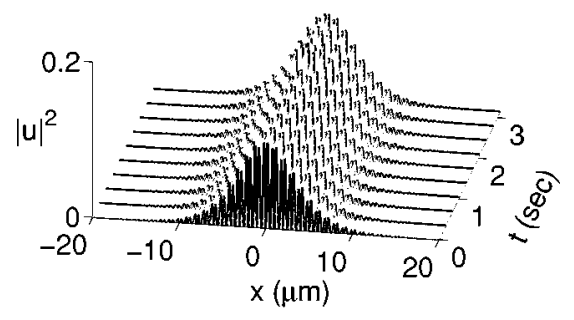

(b)

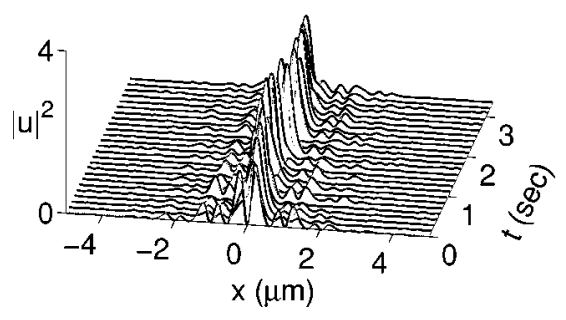

FIG. 11. Evolution (a) of a stable lattice soliton with $E=9.55$ and (b) of an unstable lattice soliton with $E=10$, for $V_{0}=10, L$ $=1 \mu \mathrm{m}$ in ${ }^{87} \mathrm{Rb}$ condensates. Both eigenenergies reside in the second band gap of the dispesion curve.

eral approximate procedures have been developed to approximate the solutions of a nonlinear Schrödinger equation with a periodic potential. Two of these models are extensively used in the literature: the tight-binding approximation and the coupled-mode theory [34]. We will illustrate how these models are obtained and discuss about the regimes of their validity. In both cases the starting point is the nonlinear Schrödinger equation with a periodic potential,

$$
i \frac{\partial u}{\partial T}=-\frac{1}{2} \frac{\partial^{2} u}{\partial X^{2}}+F(x) u+\sigma|u|^{2} u,
$$

where, for the description of optically induced lattices in Bose-Einstein condensates, $F(x)=V_{0} \sin ^{2}(\pi X / 2 D)$, and $D$ is the period of the lattice.

\section{A. Tight-binding approximation}

The tight-binding approximation [34] is extensively used to describe solitons in periodic lattices. It provides a simplified model that can provide accurate results when the original assumptions are valid. Here, we compare this model with the mean-field equation and present necessary conditions for the regimes of its validity.

We first assume that the Floquet-Bloch modes $v$ are highly confined into the lattice. This basic assumption in the tight-binding approximation implies that only the first band of the complete band structure is considered and allows the decomposition of the wave function $u$ into a sum of local modes, $\phi_{n}$. Each of these local modes corresponds to the lowest order linear eigenfunction of each potential in isolation, which is located at the minimum of the $n$th potential well. Thus, $\phi$ satisfies the linear eigenvalue problem, 


$$
E \phi=-\frac{1}{2} \frac{\partial^{2} \phi}{\partial X^{2}}+F_{0}(x) \phi
$$

where $F_{0}(X)$ is given by $F_{0}(X)=V_{0} \sin ^{2}(\pi X / 2 D)$ when $|X|<1, F_{0}(X)=V_{0}$ for $|X|>1$, and $E$ is the corresponding eigenvalue. An alternative base can be that of a Wannier function [35]. Using $\phi$ as the building element of the expansion, one can write

$$
u(x, t)=\sum_{n} c_{n}(T) \phi_{n}(X) \exp (-i E T),
$$

where $\phi_{n}(X)=\phi(X-n D)$. We substitute Eq. (14) into Eq. (12), multiply with $\phi_{m}$, and integrate over $x$. The resulting equation reads

$$
\begin{aligned}
i \sum_{n}\left\langle\phi_{m} \mid \phi_{n}\right\rangle \dot{c}_{n}+E \sum_{n \neq m}\left\langle\phi_{m} \mid \phi_{n}\right\rangle c_{n} \\
=-\frac{1}{2} \sum_{n \neq m}\left\langle\phi_{m} \mid \phi_{n X X}\right\rangle c_{n}+\sigma \sum_{n}\left\langle\phi_{m} \mid \phi_{n}^{3}\right\rangle\left|c_{n}\right|^{2} c_{n} \\
\quad+\left\langle\phi_{m} \mid \Delta F_{m}(X) \phi_{m}\right\rangle c_{m}+\sum_{n \neq m}\left\langle\phi_{m} \mid F(X) \phi_{n}\right\rangle c_{n},
\end{aligned}
$$

where we made use of the Dirac notation, $\left\langle\phi_{m} \mid \phi_{n}\right\rangle$ $=\int \phi_{m}^{*} \phi_{n} d X, \quad \Delta F_{m}=F(X)-F_{0}(X-D m), \quad$ and $\phi_{m X}$ $=\partial \phi_{m} / \partial X$. Each eigenfunction of Eq. (13), which belongs in the discrete spectrum, decays exponentially outside the potential with a rate of decay equal to $k_{i}^{2}=2\left(V_{0}-E\right)$. Thus, the coupling between second neighbors will be much smaller compared to the first-order coupling. Notice that the tightbinding approximation is valid when $k_{i}^{2} \gg 1$, and so

$$
\left\langle\phi_{m} \mid \phi_{(m \pm 1) X X}\right\rangle \approx k_{i}^{2}\left\langle\phi_{m} \mid \phi_{m \pm 1}\right\rangle \gg\left\langle\phi_{m} \mid \phi_{m \pm 1}\right\rangle .
$$

Then, normalizing $\phi_{n}$ such that $\left\langle\phi_{n} \mid \phi_{n}\right\rangle=1$, Eq. (15) becomes

$$
i \dot{c}_{n}=V c_{n}-\kappa\left(c_{n+1}+c_{n-1}\right)+\gamma\left|c_{n}\right|^{2} c_{n},
$$

which is the usual form of the tight-binding approximation. Equation (17) is in agreement with the results obtained in Ref. [13]. In Eq. (17), $V=(1 / 2)\left\langle\phi_{m} \mid \phi_{m X X}\right\rangle$ $+\left\langle\phi_{m} \mid \Delta F_{m}(X) \phi_{m}\right\rangle$ is a shift in the eigenvalue due to the perturbation in the original potential, $\kappa$ $=(1 / 2)\left\langle\phi_{m} \mid \phi_{(m \pm 1) X X}\right\rangle+\left\langle\phi_{m} \mid F(X) \phi_{m \pm 1}\right\rangle$ is the coupling coefficient between adjacent wells, and $\gamma=\sigma\left\langle\phi_{m} \mid \phi_{m}^{3}\right\rangle$ is the effective nonlinearity. Equation (17) is known as the discrete nonlinear Schrödinger equation (DNLS). It is instructive to discuss the regime where the approximation applies.

In the tight-binding model it is assumed that the atom density is highly confined into each potential minimum that, in turn, implies that the potentials have to be deep enough. It is in this regime that the Floquet-Bloch modes of the first band can be described by the discrete model. On the other hand, if the potentials are deep, they might also have more modes (discrete eigenfunctions) that are not accounted (only the zero-node mode is considered).

Nonlinear corrections are not considered in the form of the eigenmodes of Eq. (13). These corrections become significant in the case of a high-density condensate.

Higher order bands are not taken into account. As a result, in the tight-binding description, the in-phase lattice solitons in attractive condensates and the $\pi$ out-of-phase lattice solitons in repulsive condensates share exactly the same properties in the DNLS lattice [using the transformation $c_{n}$ $\rightarrow(-1)^{n} c_{n}$ along with $T \rightarrow-T$, the $\pi$ out-of-phase solution transforms into the in-phase mode], such that, for example, they both exist in a semi-infinite band gap. In the focusing regime (when the condensate is attractive), lattice solitons do exist in a semi-infinite band gap. However, in the defocusing regime (i.e., when the condensate is repulsive) lattice solitons can be found in the finite band gap between the first and the second band, and thus, the eigenvalue is located in a bounded domain. This has several implications. As the eigenvalue increases inside the band gap, the Floquet-Bloch modes become mixed, and thus Eq. (14) fails to describe the form of the solution. This is why the rate of decay of the soliton tails, $E=2 \kappa \cosh \left(k_{i}\right)$, does not globally agree with Eq. (9).

In expansion (14), $\phi_{n}$ is always in phase (with zero phase difference along $x$ ). On the other hand, in a periodic lattice the phase varies continuously along $x$. As a consequence, it was shown in Ref. [25] that the lattice solitons exhibit transport anomalies that depend on their initial momentum within the Brillouin zone as compared with the tight-binding model.

Finally, different types of solutions [such as those shown in Figs. 9 and 10], do not have equivalence in the tightbinding description, due to the constraints of the original expansion (14).

\section{B. Coupled-mode theory}

Wave propagation close to a gap resonance of a periodically modulated lattice has been studied extensively using coupled-mode theory. According to the coupled-mode description, the wave function $u$ is expanded in Fourier modes as

$$
u=\sum_{n} u_{n} \exp [-i E T+i k n X]
$$

where $k=\pi / 2 D$. Assuming that the potential is relatively shallow, only the $n= \pm 1$ terms of Eq. (18) are important, and thus, $u$ can be expanded into a forward and a backward wave,

$$
u=u_{f} \exp [-i(E T-k X)]+u_{b} \exp [-i(E T+k X)]
$$

Substituting Eq. (19) into Eq. (12), keeping the terms that resonate at the edge of first Brillouin zone (first band gap), and assuming that the material dispersion is small (broad solutions), results into the coupled-mode equations [22], 


$$
\begin{aligned}
& i\left(\frac{\partial u_{f}}{\partial T}+c \frac{\partial u_{f}}{\partial X}\right)+\kappa u_{b}+\sigma\left(\left|u_{f}\right|^{2}+2\left|u_{b}\right|^{2}\right) u_{f}=0, \\
& i\left(\frac{\partial u_{b}}{\partial T}-c \frac{\partial u_{b}}{\partial X}\right)+\kappa u_{f}+\sigma\left(\left|u_{b}\right|^{2}+2\left|u_{f}\right|^{2}\right) u_{b}=0,
\end{aligned}
$$

where $E=V_{0} / 2+(\pi / 2 D)^{2}, \quad c=\pi / D$, and $\kappa=V_{0} / 4$. We would like to mention that, due to that the sinusoidal form of the potential of the optically induced lattice and the expansion into one forward and one backward wave, coupledmode theory can only be applied in the first band gap. The dispersion relation of Eqs. (20) and (21) can be obtained by assuming plane-wave solutions of the form $u_{f, b} \propto \exp (i \lambda X$ $-i \omega T)$, and is given by $\lambda^{2}=\left(\omega^{2}-\kappa^{2}\right) / c^{2}$. Notice that gap solitons that reside outside the edge of the Brillouin zone can also be considered by Eqs. (20) and (21) by assuming an additional phase tilt between the forward and the backward wave.

Clearly, the original expansion (19) in the coupled-mode equation is accurate as long as higher order terms excluded from Eq. (18) are not very important. This is true when the potentials are relatively shallow, and thus, Eq. (19) represents the Floquet-Bloch modes inside the band gap (see the Appendix). On the other hand, when the potential is deep, higher order terms of expansion (18) become important and can no longer be ignored. Higher order corrections to the coupled-mode equation have also been studied in the literature [36]. However, the resulting equations become rather cumbersome even by considering first-order corrections.

Finally, the linear dispersion relation of Eqs. (20) and (21) is hyperbolic and centered at the edge of the first Brillouin zone, whereas, the dispersion curve of Eq. (12) (outside the band gaps) is periodic in $k$. As a result, the coupled-mode equations can be applied in the regime where this hyperbolic approximation is accurate.

\section{CONCLUSIONS}

We have systematically studied the properties of lattice solitons in Bose-Einstein condensates with either attractive or repulsive atom interactions. This was done by exactly solving the mean-field Gross-Pitaevskii equation in the presence of a periodic potential. We have found other families of lattice soliton solutions, which are characterized by the position of the energy eigenvalue within the associated band structure. These include lattice solitons in attractive and repulsive condensates that exist in finite or semi-infinite gaps as well as nonlinear modes that exhibit atomic population cutoffs.

\section{APPENDIX: PLANE-WAVE METHODS}

The eigenvalue problem of Eq. (8) can be written as

$$
E v=-\frac{1}{2} \frac{\partial^{2} v}{\partial X^{2}}+F(X) v
$$

where $F(x)$ is a periodic potential with period $D$. To solve Eq. (A1), we make use of the periodicity of the potential and expand it as

$$
F(X)=\sum_{n} U_{G_{n}} \exp \left(i G_{n} X\right)
$$

where $G_{n}=2 \pi n / D$ are the reciprocal lattice vectors. Assuming that the lattice is finite, and using periodic boundary conditions, we can expand the solution $F(X)$ as

$$
v=\sum_{n} C_{k_{n}} \exp \left(i k_{n} X\right)
$$

where $k_{n}=2 \pi n / a$, and $a$ is the length of the lattice. After these substitutions, Eq. (A1) reduces to

$$
\left(\frac{k^{2}}{2}-E\right) C_{k}+\sum_{G} C_{k-G} U_{G}=0
$$

Solving Eq. (A4) the band structure and the corresponding Floquet-Bloch modes can be computed. The accuracy of the method depends on the number of plane waves considered in the expansion, as well as on the form and the depth of the potential. In Bose-Einstein condensates, the potential (created by the interference of laser beams, is given by $F(X)$ $=V_{0} \sin ^{2}(\pi X / D)$. In this case, only three coefficients of Eq. (A2) will be nonzero, namely, $U_{0}=V_{0} / 2$ and $U_{G_{1}}=U_{-G_{1}}$ $=-V_{0} / 4$. Thus, when the potential is relatively shallow, the defect modes between the first and the second bands can be accurately described by keeping only two terms of the expansion,

$$
\begin{gathered}
{\left[\frac{k^{2}}{2}+U_{0}-E\right] C_{k}+U_{G_{1}} C_{k-G_{1}}=0,} \\
{\left[\frac{\left(k-G_{1}\right)^{2}}{2}+U_{0}-E\right] C_{k-G_{1}}+U_{0} C_{k}=0 .}
\end{gathered}
$$

Inside the first band gap, the lattice momentum will, in general, be complex, $k=\pi / D+i k_{i}$. The resulting compatibility condition of Eqs. (A5) and (A6) reads

$$
\left[\frac{1}{2}\left(\frac{\pi}{D}\right)^{2}+\frac{V_{0}}{2}-\frac{k_{i}^{2}}{2}-E\right]^{2}+\left(\frac{k_{i} \pi}{D}\right)^{2}=\left(\frac{V_{0}}{4}\right)^{2}
$$

By solving Eq. (A7), one can find the rate of the decay of the soliton tails,

$$
k_{i}^{2}=-2 E-\frac{\pi^{2}}{D^{2}}+V_{0}+\frac{2 \pi}{D} \sqrt{2 E-V_{0}+\left(D V_{0} / 2 \pi\right)^{2}}
$$

The value of $E$ inside the band gap where the fastest decay of the defect mode is achieved can be found by setting $\partial k_{i} / \partial E=0$ and is given by $E=\pi^{2} / 2 D^{2}+V_{0} / 2$ $-D^{2} V_{0}^{2} / 32 \pi^{2}$. Furthermore, the form of a defect eigenmode inside the band gap is 


$$
v=\left[\cos \frac{\phi}{2} \cos \frac{\pi X}{D}+\sin \frac{\phi}{2} \sin \frac{\pi X}{D}\right] e^{-k_{i} X},
$$

where

$$
\phi=\arctan (\beta / \alpha)
$$

and $\alpha=\pi^{2} / 2 D^{2}+V_{0} / 2-k_{i}^{2} / 2-E, \beta=k_{i} \pi / D$. The FloquetBloch modes at the edge of the first and the beginning of the second Brillouin zone (denoted by $v_{\mp}$ ) can be found by setting $E \rightarrow \pi^{2} / 2 D^{2}+V_{0} / 4$ or $E \rightarrow \pi^{2} / 2 D^{2}+3 V_{0} / 4, k_{i}^{2} \rightarrow 0$, and taking into account that $\phi$ is a continuous function. In these two limits, $\phi \rightarrow 0, \pi$, and the Floquet-Bloch mode are given by

$$
v_{-}=\cos (\pi X / D), v_{+}=\sin (\pi X / D) .
$$

Equation (A9) can then be written as a linear superposition of the Floquet-Bloch mode $v_{\mp}(X)$,

$$
v=\left[v_{-}(X) \cos \frac{\phi}{2}+v_{+}(X) \sin \frac{\phi}{2}\right] e^{-k_{i} X} .
$$

As $E$ increases inside the band gap, $\phi$ also increases from 0 to $\pi$, and the form of the corresponding defect mode is directly affected by the position of the eigenvalue inside the band gap. More specifically, when the eigenvalue is close to the first (second) band, the periodic modulations will have a cosinusoidal (sinusoidal) form, whereas, in between they will be mixed with coefficients that are determined by the position of the eigenvalue inside the band gap.
[1] M. BenDahan, E. Peik, J. Reichel, Y. Castin, and C. Salomon, Phys. Rev. Lett. 76, 4508 (1996).

[2] B.P. Anderson and M.A. Kasevich, Science 282, 1686 (1998).

[3] C. Orzel, A.K. Tuchman, M.L. Fenselau, M. Yasuda, and M.A. Kasevich, Science 291, 2386 (2001).

[4] O. Morsch, J.H. Müller, M. Cristiani, D. Ciampini, and E. Arimondo, Phys. Rev. Lett. 87, 140402 (2001).

[5] F.S. Cataliotti, S. Burger, C. Fort, P. Maddaloni, F. Minardi, A. Trombettoni, A. Smerzi, and M. Inguscio, Science 293, 843 (2001).

[6] S. Burger, F.S. Cataliotti, C. Fort, F. Minardi, M. Inguscio, M.L. Chiofalo, and M.P. Tosi, Phys. Rev. Lett. 86, 4447 (2001).

[7] Yu.B. Ovchinnikov, J.H. Müller, M.R. Doery, E.J.D. Vredenbregt, K. Helmerson, S.L. Rolston, and W.D. Phillips, Phys. Rev. Lett. 83, 284 (1999).

[8] M. Kozuma, L. Deng, E.W. Hagley, J. Wen, R. Lutwak, K. Helmerson, S.L. Rolston, and W.D. Phillips, Phys. Rev. Lett. 82, 871 (1999).

[9] L. Deng, E.W. Hagley, J. Denschlag, J.E. Simsarian, M. Edwards, C.W. Clark, K. Helmerson, S.L. Rolston, and W.D. Phillips, Phys. Rev. Lett. 83, 5407 (1999).

[10] M. Cristiani, O. Morsch, J.H. Müller, D. Ciampini, and E. Arimondo, Phys. Rev. A 65, 063612 (2002).

[11] K. Berg-Sørensen and K. Mølmer, Phys. Rev. A 58, 1480 (1998).

[12] D.I. Choi and Q. Niu, Phys. Rev. Lett. 82, 2022 (1999).

[13] A. Trombettoni and A. Smerzi, Phys. Rev. Lett. 86, 2353 (2001).

[14] F.Kh. Abdullaev, B.B. Baizakov, S.A. Darmanyan, V.V. Konotop, and M. Salerno, Phys. Rev. A 64, 043606 (2001).

[15] J.C. Bronski, L.D. Carr, B. Deconinck, J.N. Kutz, and K. Promislow, Phys. Rev. E 63, 036612 (2001).

[16] J.C. Bronski, L.D. Carr, R. Carretero-Gonzalez, B. Deconinck, J.N. Kutz, and K. Promislow, Phys. Rev. E 64, 056615 (2001).

[17] J.C. Bronski, L.D. Carr, B. Deconinck, and J.N. Kutz, Phys. Rev. Lett. 86, 1402 (2001).
[18] B. Wu, R.B. Diener, and Q. Niu, Phys. Rev. A 65, 025601 (2002).

[19] D. Diakonov, L.M. Jensen, C.J. Pethick, and H. Smith, Phys. Rev. A 66, 013604 (2002).

[20] D.N. Christodoulides and R.I. Joseph, Phys. Rev. Lett. 62, 1746 (1989).

[21] A.B. Aceves and S. Wabnitz, Phys. Lett. A 141, 37 (1989).

[22] H.G. Winful, Appl. Phys. Lett. 46, 527 (1985).

[23] D.N. Christodoulides and R.I. Joseph, Opt. Lett. 13, 794 (1988).

[24] H.S. Eisenberg, Y. Silberberg, R. Morandotti, A.R. Boyd, and J.S. Aitchison, Phys. Rev. Lett. 81, 3383 (1998).

[25] N.K. Efremidis, S. Sears, D.N. Christodoulides, J.W. Fleischer, and M. Segev, Phys. Rev. E 66, 046602 (2002).

[26] J.W. Fleischer, N.K. Efremidis, T. Carmon, D.N. Christodoulides, and M. Segev, Opt. Photonics News 13, 49 (2002).

[27] J.W. Fleischer, T. Carmon, M. Segev, N.K. Efremidis, and D.N. Christodoulides, Phys. Rev. Lett. 90, 023902 (2003).

[28] J.W. Fleischer, M. Segev, N.K. Efremidis, and D.N. Christodoulides, Nature (London) 422, 147 (2003).

[29] D. Mandelik, H.S. Eisenberg, Y. Silberberg, R. Morandotti, and J.S. Aitchison, Phys. Rev. Lett. 90, 053902 (2003).

[30] P.J.Y. Louis, E.A. Ostrovskaya, C.M. Savage, and Y.S. Kivshar, Phys. Rev. A 67, 013602 (2003).

[31] E.P. Gross, Nuovo Cimento 20, 454 (1961); E.P. Gross, J. Math. Phys. 4, 195 (1963).

[32] L.P. Pitaevskii, Zh. Éksp. Teor. Fiz. 40, 646 (1961) [Sov. Phys. JETP 13, 451 (1961)].

[33] C. Kittel, Introduction to Solid State Physics (Wiley, New York, 1986).

[34] In optics, the tight-binding approximation is also called coupled-mode theory. To avoid confusion between the coupled- mode theory used for discrete lattices and for Bragg resonances we will call the first one tight-binding approximation.

[35] G.L. Alfimov, P.G. Kevrekidis, V.V. Konotop, and M. Salerno, Phys. Rev. E 66, 046608 (2002).

[36] Takeshi Iizuka and C. Martijn de Sterke, Phys. Rev. E 62, 4246 (2000). 